\title{
Experimental Study to Develop a Method for Improving Sample Collection to Monitor Laryngoscopes after Reprocessing
}

\author{
Savina Ditommaso, Monica Giacomuzzi, Elisa Ricciardi and Carla Zotti \\ Department of Public Health and Pediatrics, University of Turin, Turin, Italy
}

Background/Aims: The microbiological surveillance of endoscopes and automated flexible endoscope reprocessing have been proven to be two of the most difficult and controversial areas of infection control in endoscopy. The purpose of this study was to standardize a sampling method for assessing the effectiveness of standard reprocessing operating procedures for flexible fiberoptic laryngoscopes (FFLs).

Methods: First, the sampling devices were directly inoculated with Bacillus atrophaeus spores; second, tissue non tissue (TNT) wipes were tested on artificially contaminated surfaces and on FFLs.

Results: Comparison of the sponges, cellulose, and TNT wipes indicated that the TNT wipes were more effective in releasing spores (93\%) than the sponges (49\%) and cellulose wipes (52\%). The developed protocol provides a high efficiency for both collection and extraction from the stainless steel surface ( $87 \%$ of the spores were removed and released) and from the FFL ( $85 \%$ of the spores were removed and released), with relatively low standard deviations for recovery efficiency, particularly for the analysis of the FFL.

Conclusions: TNT wipes are more efficient for sampling surface areas, thereby aiding in the accuracy and reproducibility of environmental surveillance. Clin Endosc 2018;51:463-469

Key Words: Flexible fiberoptic laryngoscope; Disinfection; Sampling; Wipes

\section{INTRODUCTION}

Hospital-acquired infections are frequently a result of invasive procedures or are caused by bacteria present in patients' surroundings. The control of disinfection and/or sterilization systems is a guarantee of quality and must be performed using standardized methods. Surface sampling is performed on a frequent basis in all situations where clean environment monitoring is required, e.g., in healthcare facilities, veterinary fields, and pharmaceutical and food industries.

Received: December 21, 2017 Revised: April 10, 2018

Accepted: April 10, 2018

Correspondence: Savina Ditommaso

Department of Public Health and Pediatrics, University of Turin, Piazza Polonia, 94, Turin 10126, Italy

Tel: +39-011-670-5841, Fax: +39-011-670-5881, E-mail: savina.ditommaso@unito.it ORCID: https://orcid.org/0000-0003-1590-9009

(c) This is an Open Access article distributed under the terms of the Creative Commons Attribution Non-Commercial License (http://creativecommons.org/ licenses/by-nc/3.0) which permits unrestricted non-commercial use, distribution, and reproduction in any medium, provided the original work is properly cited.
In the most frequently employed methods, moistened swabs, sponges, or sterile gauzes are wiped over the surfaces being studied and are then inoculated in different culture media, with or without prior enrichment. Another method uses a polystyrene contact plate, which is similar to a small Petri dish with a convex surface that is pressed onto any flat surface to obtain a sample. ${ }^{1}$

Traditional surface sampling methods utilizing swabs and wipes have been plagued by poor recovery and highly variable results, ${ }^{2}$ with reported recovery efficiencies ranging from less than $1 \%{ }^{3}$ to greater than $90 \%,{ }^{4}$ depending on the experimental conditions.

An ideal sampling method should satisfy three important criteria: first, the sampler must effectively remove bacteria from the surface being sampled; second, removal should maximize bacterial collection and minimize bacterial damage; and finally, the sampler must release as much of the captured bacteria as possible for analysis. ${ }^{2}$ The sampling method should also be selected to complement the analytical technique (e.g., 
culture-based vs polymerase chain reaction-based).

Our knowledge of the most effective sampling methods and our level of confidence in the results obtained by wiping, swabbing, and other sample collection strategies are still limited. Several studies ${ }^{5-9}$ have reported the efficiency of different types of sampling devices (e.g., electrostatic wipes, one-ply composite tissue, sponges, cotton-tipped swabs, and calcium alginate swabs) used for the quantitative recovery of different infectious agents from environmental surfaces. These studies have highlighted that different devices exhibit varying levels of performance and indicated that the wipe-rinse technique has a significantly higher recovery efficiency than other methods. $^{10}$

Indeed, even if conventional hygiene swabbing is widely used, this method has been reported to recover only a small proportion of the total bacterial population present on a surface. The surface area that can be sampled by a swab is relatively small; the method is designed for high levels of microbial contamination; and there is a poor correlation among investigators as to the amount of microbial contamination removed and recovered from swabs. ${ }^{11}$

Flexible endoscopes cannot withstand the high temperatures and pressure changes in autoclave cycles and hence are usually decontaminated by cleaning followed by high-level disinfection (HLD) with a sterilant. Sampling is a critical component of the investigation after reprocessing of flexible endoscopes.

However, although there are shared protocols for sampling $^{12-14}$ for channeled instruments, such as flexible gastrointestinal endoscopes, there are no precise indications for instruments without channels. Moreover, there are several disparities among published studies regarding sampling methods for microbiological monitoring: in some studies, samples were obtained using swab cultures, ${ }^{15-17}$ whereas in others, flexible endoscopes subjected to surveillance were dipped into a sterile culture medium which was then submitted for microbiological analysis. ${ }^{18,19}$ However, these studies did not provide guidance regarding the specific sampling method used as a reference.

Therefore, the purpose of this study is (1) to assess the ability of sampling devices to collect and detect low levels of bacteria from surfaces and (2) to validate a sampling method for assessing the effectiveness of standard reprocessing operating procedures for flexible fiberoptic laryngoscopes (FFLs).

\section{MATERIALS AND METHODS}

\section{Test run stages}

A series of experiments were conducted to develop a final sampling method protocol. In the first stage of the study, we compared the recovery of Bacillus atrophaeus spores that were inoculated directly on three devices to evaluate the extraction efficiency of each sampling device. In the second stage of the study, the efficacy of the selected sampling material was tested on artificially contaminated surfaces and on FFLs.

\section{Test organism}

As suggested by the ISO $11737,{ }^{20}$ the microorganisms selected as the test agent were the spores of an aerobic bacteria that can persist during the drying stage; the inoculum was a $40 \%$ ethanol suspension of B. atrophaeus (ATCC 9372) at a concentration of $2.9 \times 10^{7} / 0.1 \mathrm{~mL}$ (Mesa labs, Bozeman, MT, USA).

\section{First stage: extraction efficiency}

We determined the extraction efficiency of the bacterial transfer to the extraction solution from three collection materials, e.g., cellulose wipes (Napkin, Ravenna, Italy), tissue non tissue (TNT) wipes (a blend of cellulose and synthetic fibers), and $3 \mathrm{M}^{\mathrm{TM}}$ hydrated sponge with $10-\mathrm{mL}$ neutralizing buffer (3M, Milano, Italy) (Fig. 1).

We selected these sampling devices because they can be rubbed over surfaces and are highly absorptive in contrast with stick swabs that can be used to sample hard-to-reach small areas (e.g., inside instrument channel).

A vortexing method for extracting spores from the wipes and from the sponges was evaluated using the following method. Ten cellulose wipes and 10 TNT wipes were cut to measure $8.0 \times 6.0 \mathrm{~cm}$, sterilized by autoclaving, and placed into 50 -mL tubes; 20 wipes $(10+10)$ and 10 hydrated sponges (10 $\mathrm{mL}$ of buffered peptone water broth in a sample bag) were directly inoculated with $10^{3}$ colony-forming units (CFU) of the spore suspension. Following inoculation, we added $30 \mathrm{~mL}$ of Page's buffer into 50-mL tubes and $20 \mathrm{~mL}$ of Page's buffer into the sponge sample bag. Ten additional tubes with $30 \mathrm{~mL}$ of Page's buffer were added with $10^{3} \mathrm{CFU}$ of the spore suspen-

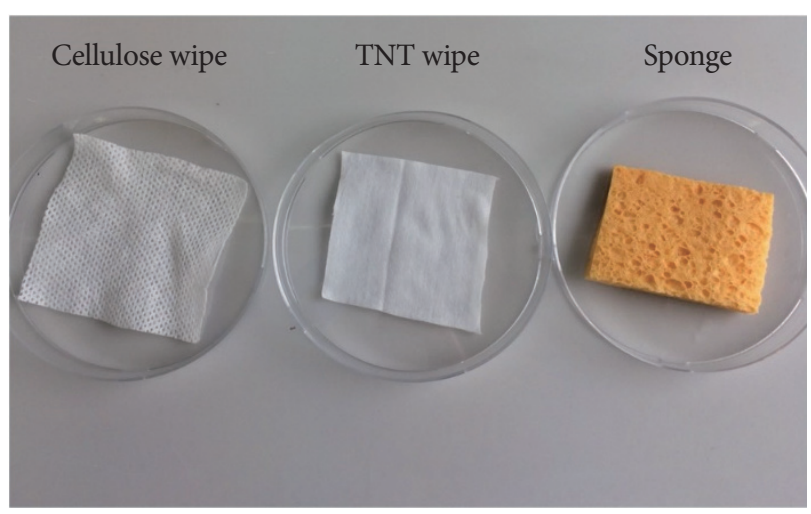

Fig. 1. Photograph of the three sampling devices. TNT, tissue non tissue. 
sion as references.

Spores were extracted from the wipes by vortexing for 30 $s$ and from the sponges using a Stomacher ${ }^{\circledR} 400$ Circulator (VWR International, Radnor, PA, USA) and massaging for 1 $\mathrm{min}$ at $200 \mathrm{rpm}$. A 1-mL aliquot of the extraction suspension and reference suspension were spread onto a Petrifilm nutrient agar medium (Oxoid S.p.A., Milano, Italy) in triplicate and then incubated at $30^{\circ} \mathrm{C}$ for $24-48 \mathrm{~h}$. Only the plates with counts between 30 and $300 \mathrm{CFU}$ were included.

\section{Second stage: recovery efficiency}

The recovery efficiency was calculated; two surfaces were seeded with the B. atrophaeus spore suspension: a stainless steel reference disc and an FFL (ENF Type GP; Olympus, Tokyo, Japan). This assessment was based on the methods described in the European standard for chemical disinfectants and antiseptics (EN 14347, EN 13704) $)^{21,22}$ and the ISO 117371 standard ${ }^{20}$ that specifies the requirements to be met in the determination of bioburden. This standard suggests using low levels of microorganisms for the validation of the method. According to this norm, it is not possible to enumerate the bioburden exactly nor define a single technique to be used in all situations for the removal of microorganisms in preparation for enumeration. The guidance given in the informative annexes provides explanations and methods that are regarded as suitable means for complying with the requirements. Methods other than those given in the guidance may be used if they are effective in complying with the requirements of this part of the ISO 11737.

\section{Stainless steel surface}

Ten stainless steel discs $(2.0 \mathrm{~cm})$ were directly inoculated with $0.1 \mathrm{~mL}$ of the decimal dilution (containing approximately $10^{3}$ viable spores) of the stock suspension and placed to dry for $10 \mathrm{~min}$ in a microbiological safety cabinet. Spores were collected using aseptic procedures (wearing sterile gloves) from the sample discs by moistening a sterile TNT wipe with 0.5 $\mathrm{mL}$ of sterile water, wiping the surface with single horizontal strokes to the left and right, folding the exposed side to the interior, and wiping with single upward and downward strokes. Spores were collected by the same individual to exclude the impact of possible man-made interference. Thereafter, the wipe was placed in a prelabelled tube containing $20 \mathrm{~mL}$ of sterile Page's buffer and extracted by vortexing for $30 \mathrm{~s}$. One milliliter of the extraction suspension was spread onto a Petrifilm nutrient agar medium in triplicate and then incubated at $30^{\circ} \mathrm{C}$ for $24-48 \mathrm{~h}$. Ten additional discs were inoculated with $10^{3}$ viable spores as references. The spore was removed from the reference discs' surfaces by vortexing with glass beads and cultured for the enumeration of the CFU as described above.

\section{FFL surface}

After disinfection of the FFL, the area $24 \mathrm{~cm}$ from the distal end of the endoscope insertion tube was inoculated with 0.2 $\mathrm{mL}\left(1.5 \times 10^{3} \mathrm{CFU}\right)$ of the diluted spore suspension and placed to dry for $10 \mathrm{~min}$ in a microbiological safety cabinet. Spores were collected using aseptic procedures from the FFL with a sterile TNT wipe pre-moistened with $0.5 \mathrm{~mL}$ of sterile water by wiping the surface with single vertical strokes from top to bottom of the exterior of the insertion tube, folding the exposed side of the wipe to the interior, and wiping once again (Fig. 2).

The procedure was conducted 10 times, in which the instrument was disinfected each time. The wipe was placed in a prelabelled tube containing $20 \mathrm{~mL}$ of sterile Page's solution, and spores were extracted by vortexing for $30 \mathrm{~s}$ for enumeration by culture. A 1-mL aliquot of the extraction suspension was spread onto a Petrifilm nutrient agar medium in triplicate and then incubated at $30^{\circ} \mathrm{C}$ for $24-48 \mathrm{~h}$.

\section{Calculations}

The extraction efficiency was calculated as the mean CFU obtained from the extraction suspension from the sponges, cellulose wipes, and TNT wipes relative to the mean CFU obtained from the reference suspensions.

The recovery efficiency was calculated as the mean CFU obtained from the TNT wipe samples relative to the mean CFU obtained from the reference culture suspensions.

Standard deviations (SDs) and 95\% confidence intervals (CIs) were calculated. One-way layout analysis of variance (ANOVA) was conducted to compare the means of the three sampling devices (extraction performance).

Repeatability was calculated in accordance with the ISO $13843 ;^{23}$ the formula was as follows: $r=2.8 \times s_{r}$, where $s_{\mathrm{r}}$ is the repeatability $\mathrm{SD}$.

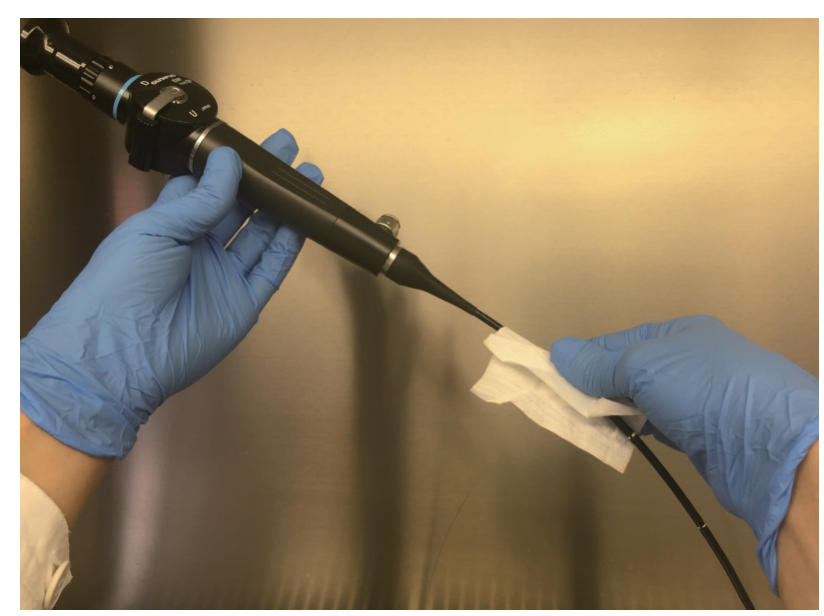

Fig. 2. Spore collection from the flexible fiberoptic laryngoscope surface using a sterile tissue non tissue wipe. 


\section{RESULTS}

\section{Extraction efficiency}

Extraction performance varied significantly across the sampling devices, indicating that the material has an impact on the release of spores from the devices into the extraction solution. The TNT wipes yielded the best recovery rates (93\%), while the cellulose wipes and sponges yielded lower recovery rates of $49 \%$ and 52\%, respectively (Fig. 3).

The difference in the recovery efficiency between the TNT wipes and the other devices suggests that the TNT wipes are significantly more efficient than the other two sampling devices in terms of recovery (ANOVA: $f$-ratio, 28.07341; $p<0.00001)$. Consequently, we selected these devices to proceed with the next phase of the study.

\section{Recovery efficiency from the stainless steel surfaces}

Fig. 4 shows the recovery efficiency of the TNT wipe method for each of the 10 replicates. The percentage of the recovered spores was calculated on the basis of the mean CFU of the inoculated reference discs. These data showed that the spore material removed from the carriers and cultured for the enumeration of the CFU was satisfactory (mean recovery, $87 \%$; SD, 10.3 ; $95 \%$ CI, $81 \%$ to $93 \%$; repeatability, 0.15 ) and confirmed the results obtained in the first stage of the study: once captured by wipes, microorganisms are readily released into the extraction solution.

\section{Recovery efficiency from the FFL surfaces}

The numbers of spores recovered from the FFLs using the TNT wipes are shown in Fig. 5; when B. atrophaeus spores were spotted on the instrument surfaces, the mean extraction efficiency was $85 \%$ (SD, $8.7 ; 95 \%$ CI, $80 \%$ to $90 \%$ ), and the repeatability was 0.13 .

\section{DISCUSSION}

Environmental microbiological surveillance is only one approach to reducing the risk of healthcare-acquired infections. Another key issue is the microbiological monitoring of invasive instruments, such as endoscopes, which are subject to disinfection and can be the source of infection and patient-to-patient transmission. Standardized methods for conducting environmental investigations or microbiological surveillance of medical devices (such as endoscopes) have not been published; therefore, endoscopic societies have established guidelines for proper reprocessing of endoscopes. Most guidelines recommend multiple steps consisting of pre-cleaning, cleaning, disinfecting, rinsing, drying, and storing. ${ }^{24,25}$

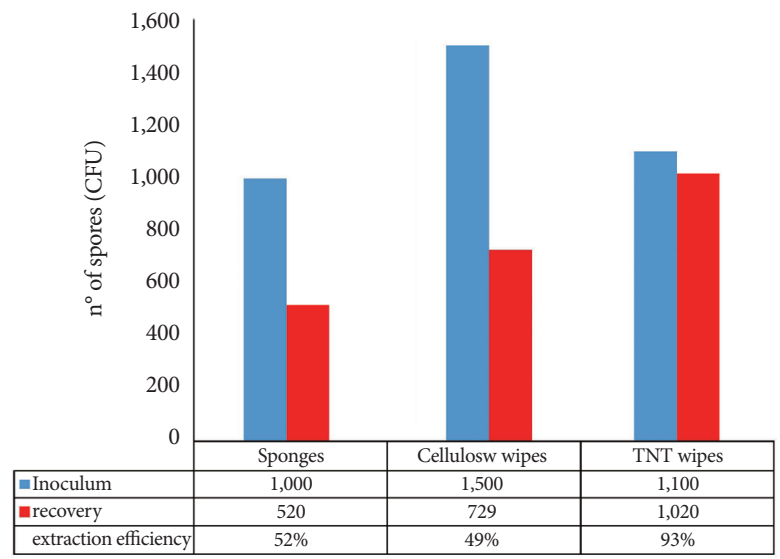

Fig. 3. Extraction efficiency among the three different sampling devices. CFU, colony-forming units; TNT, tissue non tissue.

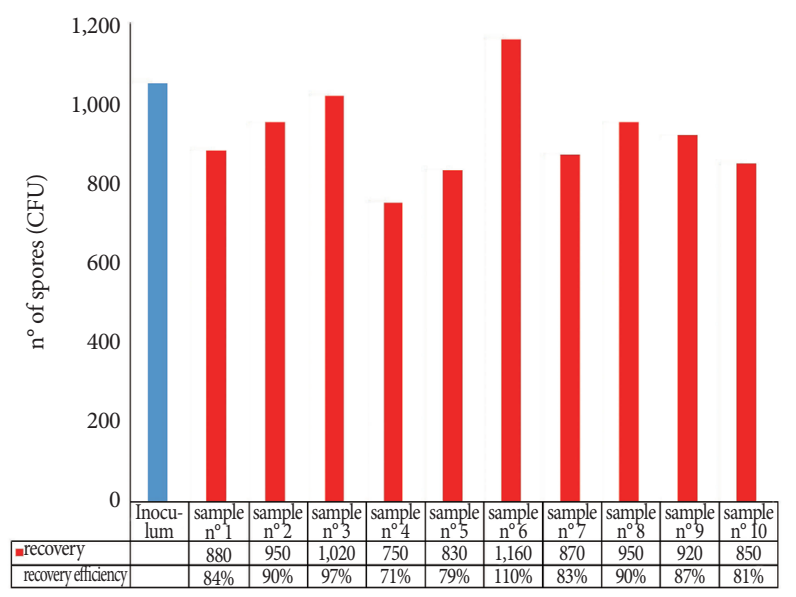

Fig. 4. Performance of the tissue non tissue wipe sampling method for the collection of Bacillus atrophaeus spores from the stainless steel surfaces. CFU, colony-forming units.

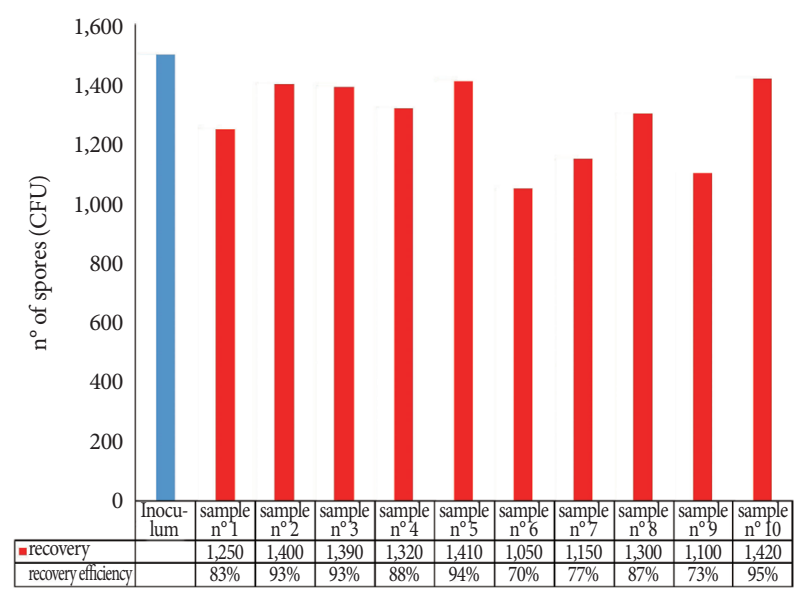

Fig. 5. Bacillus atrophaeus spore recoveries from the flexible fiberoptic laryngoscope surface. CFU, colony-forming units.

As a part of the quality control of endoscope reprocessing, microbiological monitoring of endoscopes and their related 
facilities after HLD is recommended by many organizations, such as the American Society for Gastrointestinal Endoscopy, ${ }^{24,26}$ European Society of Gastrointestinal Endoscopy, European Society of Gastroenterology and Endoscopy Nurses and Associates committee, ${ }^{26}$ and Gastroenterological Society of Australia. $^{12,27}$

Recent reports have identified carbapenem-resistant Enterobacteriaceae transmissions associated with persistently contaminated duodenoscopes for which no breaches in reprocessing were identified. ${ }^{28}$ Therefore, the Centers for Disease Control and Prevention (CDC) drafted a protocol for facilities that are considering regular monitoring to assess the adequacy of duodenoscope reprocessing. ${ }^{13}$

According to this document, the optimal frequency of surveillance cultures has not been established. International guidelines have recommended intervals ranging from every 4 weeks to annually. ${ }^{12,26} \mathrm{~A}$ facility deciding to perform surveillance cultures can consider conducting post-reprocessing cultures periodically, e.g., monthly or after every 60 procedures for each duodenoscope. Some facilities could decide to perform duodenoscope cultures weekly (e.g., after procedures on Friday to allow cultures to incubate over the weekend). Alternatively, facilities can also decide to perform cultures after reprocessing following each use. Cultures should be obtained after the duodenoscope has been reprocessed (after drying) and should include at least the instrument channel and the distal end of the duodenoscope.

However, the microbiological surveillance of endoscopes and automated flexible endoscope reprocessing have been proven to be two of the most difficult and controversial areas of infection control in endoscopy.

An interim sampling protocol developed by the CDC represents one approach to culturing of duodenoscopes. ${ }^{13}$ Facilities may use other sampling methods (e.g., flush-brush-flush method) or sample additional channels beyond those specified in this approach. The sensitivity of the interim protocol has not been determined.

FFLs are one of the most commonly used instruments in the outpatient otolaryngology setting. Unlike their channeled gastrointestinal and bronchoscope counterparts, FFLs typically do not have a working channel for biopsies and other interventions. Although it is generally considered that FFLs have a lower level of bioburden accumulation during use than their channeled counterparts, there have been reports of contamination of FFLs with body fluids, blood, and debris, such as mucus and even pathogenic organisms during routine use..$^{29}$ Even with a lower level of bioburden, FFLs have a high rate of bacterial contamination during routine clinical use. According to the Spaulding classification, ${ }^{30}$ FFLs are considered semi-critical devices and should undergo at least HLDs between uses. HLD refers to the inactivation of pathogenic organisms, such as viruses, mycobacteria, fungi, fungal spores, and, if possible, bacterial endospores. ${ }^{31,32}$

The detection of indicator organisms or pathogens on solid surfaces provides valuable risk assessment data for modelling patients' exposure to cross-infection. Ideally, the method used should permit fully reliable detection even when microorganisms are present in low numbers. Numerous studies have shown that the sampling devices (swabs, wipes, etc.) approved for environmental sampling are often awkward to use and inefficient. ${ }^{6,7}$

Recovery efficiency is dependent on the methods used for depositing bio-contaminants on surfaces, surface characteristics, wetting agents, extraction solutions, physical dissociation methods (e.g., vortex and stomacher), sampling materials, variations in sampling techniques, biological agents, and laboratory personnel. ${ }^{33,34}$

In this study, we developed a surface sampling method to detect low levels of bacteria on a nasofibroscope.

In the first stage of the study, we compared the recovery of B. atrophaeus spores inoculated directly on the three sampling devices to evaluate the efficacy of the sampling materials more commonly adapted. ${ }^{5-7,9}$

The side-by-side comparison of the sponges, cellulose wipes, and TNT wipes indicated that the TNT wipes were more effective in recovering spores (93\%) than the sponges (49\%) and cellulose wipes (52\%).

This ability to release collected organisms is probably a function of the fibrous nature of the cloth and is one of the limiting factors of their effectiveness. The tendency to retain collected organisms seen when comparing the TNT and swab/cellulose wipes indicates that swab and cellulose wipes do not release all of the collected organisms after the elution step, and this recovery of the low percentage demonstrated that the entrapped organisms were not released from the cloth. The increased recovery using the TNT wipes may also be because of the inherent antistatic coating property, which could probably aid in the release of bacteria by reducing the electrostatic discharge commonly seen in sampling devices, such as sponges and cellulose wipes.

In the second stage of the study, we tested the TNT wipes on artificially contaminated surfaces, and we designed and tested a protocol on nasofibroscopes. The applied protocol provides a high efficiency for both collection and extraction from the stainless steel surface ( $87 \%$ of the spores were removed and released) and from the FFL (85\% of the spores were removed and released), with relatively low SDs for recovery efficiency, particularly for the analysis of the FFL.

These results met the criteria required by the ISO $11737-1,{ }^{20}$ which specifies requirements and provides guidance for the 
enumeration and microbial characterization of the population of viable microorganisms on a medical device, component, raw material, or package. In fact, according to this ISO, a removal technique can be considered accurate if the recovery percentage is above $50 \%$.

The efficiency of the method described in this study provides information regarding the interpretation of the TNT wipe for environmental sample collection; these wipes identify even samples with low levels of bacterial contamination and may help in monitoring endoscopes after reprocessing, as recommended by the CDC and majority of organizations. Owing to the increasing concerns regarding antimicrobial-resistant bacteria, the CDC focused on a protocol that captures and identifies bacteria. ${ }^{13}$ This protocol has not been validated and is one approach to culturing duodenoscopes. The protocol is intended to provide facilities that are considering culturing with a starting point for a protocol that can be adapted for use. The protocol is still being developed and evaluated for the major duodenoscope types. This is an interim protocol and will be updated accordingly.

Therefore, our protocol may be used for the routine monitoring/auditing of the effectiveness of disinfection practices (hospital surface, medical device, etc.), as TNT wipes are more effective in sampling surface areas, thereby aiding in the accuracy and reproducibility of environmental surveillance.

This technique could be used in various areas of environmental microbiology where the ability to measure surface contaminations quickly, accurately, and economically is important.

\section{Conflicts of Interest}

The authors have no financial conflicts of interest.

\section{Acknowledgements}

This work was supported by Tristel Italia Srl.

\section{REFERENCES}

1. Sehulster LM, Chinn RYW, Arduino MJ, et al. Guidelines for environmental infection control in health-care facilities. Recommendations from CDC and the Healthcare Infection Control Practices Advisory Committee (HICPAC). Chicago (IL): American Society for Healthcare Engineering/American Hospital Association; 2004.

2. Edmonds JM. Efficient methods for large-area surface sampling of sites contaminated with pathogenic microorganisms and other hazardous agents: current state, needs, and perspectives. Appl Microbiol Biotechnol 2009;84:811-816

3. Buttner MP, Cruz P, Stetzenbach LD, Cronin T. Evaluation of two surface sampling methods for detection of Erwinia herbicola on a variety of materials by culture and quantitative PCR. Appl Environ Microbiol 2007;73:3505-3510
4. Edmonds JM, Collett PJ, Valdes ER, Skowronski EW, Pellar GJ, Emanuel PA. Surface sampling of spores in dry-deposition aerosols. Appl Environ Microbiol 2009;75:39-44.

5. Moore G, Griffith C. A comparison of traditional and recently developed methods for monitoring surface hygiene within the food industry: an industry trial. Int J Environ Health Res 2002;12:317-329.

6. Sanderson WT, Hein MJ, Taylor L, et al. Surface sampling methods for Bacillus anthracis spore contamination. Emerg Infect Dis 2002;8:11451151

7. Vorst KL, Todd EC, Rysert ET. Improved quantitative recovery of Listeria monocytogenes from stainless steel surfaces using a one-ply composite tissue. J Food Prot 2004;67:2212-2217.

8. Brown GS, Betty RG, Brockmann JE, et al. Evaluation of rayon swab surface sample collection method for Bacillus spores from nonporous surfaces. J Appl Microbiol 2007;103:1074-1080.

9. Downey AS, Da Silva SM, Olson ND, Filliben JJ, Morrow JB. Impact of processing method on recovery of bacteria from wipes used in biological surface sampling. Appl Environ Microbiol 2012;78:5872-5881.

10. Ruple-Czerniak A, Bolte DS, Burgess BA, Morley PS. Comparison of two sampling and culture systems for detection of Salmonella enterica in the environment of a large animal hospital. Equine Vet J 2014;46:499502.

11. Bredholt S, Maukonen J, Kujanpää K, et al. Microbial methods for assessment of cleaning and disinfection of food-processing surfaces cleaned in a low-pressure system. Eur Food Res Technol 1999;209:145152.

12. Digestive Health Foundation. Infection control in endoscopy [Internet]. Sydney: Gastroenterological Society of Australia; c2008 [updated 2008 Feb; cited 2018 Jul 11]. Available from: http://cart.gesa.org.au/membes/ files/Clinical\%20Guidelines\%20and\%20Updates/Endoscopy_Microbiological_Testing.pdf.

13. Centers for Disease Control and Prevention. Duodenoscope surveillance sampling \& culturing: reducing the risks of infection [Internet]. Atlanta (GA): CDC; c2015 [updated 2018 Feb 28; cited 2018 Jul 11]. Available from: https://www.cdc.gov/hai/organisms/cre/cre-duodenoscope-surveillance-protocol.html.

14. Ministère de la santé et des solidarités. Elements d'assurance qualite en hygiene relatifs au contrôle microbiologique des endoscopes et à la traçabilite en endoscopie [Internet]. Paris: Société Française d'Endoscopie Digestive; c2007 [cited 2018 Jul 11]. Available from: http://www.sfed.org/ professionnels/commissions-et-actions/plateaux-techniques-qualite-hygiene-et-securite/qualite/textes.

15. Bhatt JM, Peterson EM, Verma SP. Microbiological sampling of the forgotten components of a flexible fiberoptic laryngoscope: what lessons can we learn? Otolaryngol Head Neck Surg 2014;150:235-236.

16. Elackattu A, Zoccoli M, Spiegel JH, Grundfast KM. A comparison of two methods for preventing cross-contamination when using flexible fiberoptic endoscopes in an otolaryngology clinic: disposable sterile sheaths versus immersion in germicidal liquid. Laryngoscope 2010;120:2410-2416.

17. Tzanidakis K, Choudhury N, Bhat S, Weerasinghe A, Marais J. Evaluation of disinfection of flexible nasendoscopes using Tristel wipes: a prospective single blind study. Ann R Coll Surg Engl 2012;94:185-188.

18. Bhattacharyya N, Kepnes LJ. The effectiveness of immersion disinfection for flexible fiberoptic laryngoscopes. Otolaryngol Head Neck Surg 2004;130:681-685.

19. Liming B, Funnell I, Jones A, Demons S, Marshall K, Harsha W. An evaluation of varying protocols for high-level disinfection of flexible fiberoptic laryngoscopes. Laryngoscope 2014;124:2498-2501.

20. International Organization for Standardization. ISO 11737-1:2006. Sterilization of medical devices -- microbiological methods -- part 1: determination of a population of microorganisms on products [Internet]. Geneva: ISO; c2006 [updated 2006 Apr; cited 2018 Jul 11]. Available from: https://www.iso.org/standard/38711.html.

21. European Committee for Standardization. EN 14347 - Chemical dis- 
infectants and antiseptics - basic sporicidal activity - test method and requirements (phase 1, step 1). Brussels: European Standards; 2005.

22. European Committee for Standardization. EN 13704 - Chemical disinfectants - quantitative suspension test for evaluation of sporicidal activity of chemical disinfectants used in food, industrial, domestic and institutional areas - test method and requirements (phase 2, step 1). Brussels: European Standards; 2002.

23. International Organization for Standardization. ISO/TR 13843: Water quality - guidance on validation of microbiological methods [Internet]. Geneva: ISO; c2000 [updated 2000 Jun; cited 2018 Jul 11]. Available from: https://www.iso.org/standard/22599.html.

24. ASGE Quality Assurance In Endoscopy Committee, Petersen BT, Chennat J, et al. Multisociety guideline on reprocessing flexible gastrointestinal endoscopes: 2011. Gastrointest Endosc 2011;73:1075-1084.

25. Lee YK, Park JB. Steps of reprocessing and equipments. Clin Endosc 2013;46:274-279.

26. Beilenhoff U, Neumann CS, Rey JF, Biering H, Blum R, Schmidt V. ESGE-ESGENA guideline for quality assurance in reprocessing: microbiological surveillance testing in endoscopy. Endoscopy 2007;39:175181.

27. Saviuc P, Picot-Guéraud R, Shum Cheong Sing J, et al. Evaluation of the quality of reprocessing of gastrointestinal endoscopes. Infect Control Hosp Epidemiol 2015;36:1017-1023.

28. Epstein L, Hunter JC, Arwady MA, et al. New Delhi metallo-beta-lactamase-producing carbapenem-resistant Escherichia coli associated with exposure to duodenoscopes. JAMA 2014;312:1447-1455.

29. Muscarella LF. Prevention of disease transmission during flexible laryngoscopy. Am J Infect Control 2007;35:536-544.

30. Spaulding EH. Chemical disinfection and antisepsis in the hospital. J Hosp Res 1972;9:5-31.

31. Collins WO. A review of reprocessing techniques of flexible nasopharyngoscopes. Otolaryngol Head Neck Surg 2009;141:307-310.

32. McDonnell G, Burke P. Disinfection: is it time to reconsider Spaulding? J Hosp Infect 2011;78:163-170.

33. Lutz JK, Crawford J, Hoet AE, Wilkins JR 3rd, Lee J. Comparative performance of contact plates, electrostatic wipes, swabs and a novel sampling device for the detection of Staphylococcus aureus on environmental surfaces. J Appl Microbiol 2013;115:171-178.

34. Rose LJ, Hodges L, O'Connell H, Noble-Wang J. National validation study of a cellulose sponge wipe-processing method for use after sampling Bacillus anthracis spores from surfaces. Appl Environ Microbiol 2011;77:8355-8359. 